\title{
Vibrio penaeicida sp. nov., a Pathogen of Kuruma Prawns (Penaeus japonicus)
}

\author{
KATSUYA ISHIMARU, ${ }^{1}$ MASAYO AKAGAWA-MATSUSHITA, ${ }^{2}$ AND KIYOKUNI MUROGA ${ }^{1 *}$ \\ Faculty of Applied Biological Science, Hiroshima University, Higashihiroshima-shi, Hiroshima 724, ${ }^{1}$ \\ and Department of Chemistry, University of Occupational and Environmental Health, \\ Yahatanishi-ku, Kitakyushu-shi 807,2 Japan
}

\begin{abstract}
Six similar strains which were isolated as pathogens of cultured kuruma prawns (Penaeus japonicus) in Japan had characteristics of the genus Vibrio. These organisms were distinguished from previously described Vibrio species by their positive results in tests for gelatinase and lipase activities and their negative reactions in tests for arginine dihydrolase and lysine and ornithine decarboxylase activities, growth in the presence of $6 \%$ $\mathrm{NaCl}$ and at $35^{\circ} \mathrm{C}$, acid production from sucrose, utilization of D-glucuronate and propionate, and luminescence. The G+C contents of the DNAs of these organisms were 46.2 to $47.0 \mathrm{~mol} \%$. The levels of DNA relatedness among these six strains were 87 to $99 \%$ (as determined by the membrane filter method), while the levels of DNA relatedness between prawn pathogen strain $\mathrm{KH}^{\mathrm{T}}{ }^{\mathrm{T}}(\mathrm{T}=$ type strain) and members of 28 previously described Vibrio species were less than $18 \%$. The name Vibrio penaeicida sp. nov. is proposed for the prawn-pathogenic strains. The type strain is strain KH-1 (= IFO $15640=$ JCM 9123).
\end{abstract}

Penaeus japonicus (kuruma prawns) is one of the most valuable cultured aquatic animal species in Japan. Vibriosis in cultured kuruma prawns has been observed since 1980, and there have been considerable losses in the prawn industry (28). The prawns affected are characterized by the formation of brown spots (nodules) in the lymphoid organs and gills. The causative bacterium was first isolated by Takahashi et al. in 1982 (29) from diseased prawns in Yamaguchi and Kumamoto Prefectures and was classified as a member of the genus Vibrio, but the strains isolated by Takahashi et al. have been lost. Later, we isolated the pathogen which causes vibriosis of kuruma prawns in specimens from various districts in Japan. The pathogen, which we isolated, tentatively designated Vibrio species PJ (11), was studied to determine its morphological, biochemical, pathological and serological characteristics (12), and we believe that this organism is the same species described by Takahashi et al. (29). The pathogen was also isolated from apparently healthy prawns and water samples obtained from prawn culture ponds (11). In this study we determined the taxonomic position of the prawn pathogen. Below we propose the name Vibrio penaeicida sp. nov. for this organism.

\section{MATERIALS AND METHODS}

Bacterial strains. The six $V$. penaeicida strains listed in Table 1 were isolated from kuruma prawns obtained in prefectures of western Japan. These strains were isolated and subcultured on ZoBell's $2216 \mathrm{e}$ agar plates (1) at $25^{\circ} \mathrm{C}$ and were maintained in semisolid ZoBell's $2216 \mathrm{e}$ medium containing $0.5 \%$ agar at $20^{\circ} \mathrm{C}$; they were subcultured every 6 months. Untreated aged seawater obtained from the Inland Sea of Japan was used to prepare ZoBell's 2216e medium.

The reference strains used in DNA hybridization experiments are listed in Table 1. Vibrio cholerae HUFP 5004 (= NIH 35A3) is not the type strain of $V$. cholerae but was found to be closely related (level of similarity, 98\%) to type strain ATCC 14035 by DNA-DNA hybridization (7).

Morphological observations. Gram staining (Hucker modification [8]) was carried out by using 1-day cultures grown on ZoBell's 2216e agar. Cell form and flagellum arrangement were determined by using an electron microscope to observe cells that had been grown on ZoBell's $2216 \mathrm{e}$ agar for 2 days at $25^{\circ} \mathrm{C}$. The cells were suspended in $2 \%$ ammonium acetate, negatively stained with $1 \%$ uranyl acetate containing $0.4 \%$ sucrose (3), and examined with a Hitachi model

* Corresponding author. Mailing address: Fish Pathology Laboratory, Faculty of Applied Biological Science, Hiroshima University, 1-4-4 Kagamiyama, Higashihiroshima-shi, Hiroshima Prefecture, Japan 724. Phone: 0824-24-7977. Fax: 0824-22-7059.
$\mathrm{H}-600 \mathrm{~A}$ transmission electron microscope operated at an accelerating voltage of $75 \mathrm{kV}$.

Physiological and biochemical characterization. Cells from a 1-day culture on ZoBell's agar were used in all tests. The $\mathrm{NaCl}$ concentration was adjusted to $2 \%$ (wt/vol) in all test media except those containing seawater or those that were used to determine the range of $\mathrm{NaCl}$ concentrations for growth. All of the inoculated test media except the media used to determine the temperature range for growth were incubated at $25^{\circ} \mathrm{C}$.

Motility was determined by the hanging drop method (9). Luminescence was observed on ZoBell's 2216e agar and luminous medium plates (26). After 8, 16, 24 , and $48 \mathrm{~h}$ of incubation, luminescence was determined in a dark room after the investigator's eyes had adapted to the dark for $5 \mathrm{~min}$. Pigmentation was determined after 7 days of incubation on ZoBell's 2216e agar. Susceptibility to vibriostatic agent $\mathrm{O} / 129$ was determined with $10-$ and $150-\mu \mathrm{g}$ discs on nutrient agar (Difco) after $24 \mathrm{~h}$ of incubation. O/129 phosphate was obtained from Sigma Chemical Co., and the discs were prepared as described by Lee et al. (20). Fermentation of glucose was determined in Hugh-Leifson medium (Eiken Chemical Co. Ltd., Tokyo, Japan) containing $1 \%$ glucose after 7 days of incubation. Cytochrome oxidase activity was determined by the Kovács method by using test strips (Nissui Pharmaceutical Co., Ltd., Tokyo, Japan) that included tetramethyl-p-phenylenediamine dihydrochloride. The cultures were smeared on strips that had been wetted with distilled water, and the appearance of a purple color was determined after $30 \mathrm{~s}$. Catalase activity was detected by the slide method. Cells were transferred to a clean glass slide, and $3 \%$ hydrogen peroxide was added. Bubble formation was determined immediately and after 5 min. Indole production was determined in $1 \%$ (wt/vol) tryptone (Difco) after incubation for 2 days. Indole was detected by the presence of a red color after $1.0 \mathrm{ml}$ of Kovács reagent (9) was added to the medium. Nitrate reduction was determined by using $1 \%$ (wt/vol) Bacto Peptone (Difco) and $0.1 \%$ (wt/vol) potassium nitrate. The results were recorded as described by MacFaddin (22) after 2 and 5 days of incubation. The Voges-Proskauer test (Barritt's test) was performed by using 1\% (wt/vol) Bacto Peptone (Difco) and 1\% (wt/vol) $\mathrm{D}$-glucose; the results were judged by using the procedures described by MacFaddin (22) after 2 and 5 days of incubation. The production of hydrogen sulfide was determined by observing the blackening of SIM medium (Eiken) every day for 4 days. Lysine and ornithine decarboxylation and arginine dihydroxylation were determined in Møller decarboxylase base (Difco) as described by MacFaddin (22) by examining preparations every day for 7 days. Hydrolysis of $o$-nitrophenyl- $\beta$-D-galactopyranoside was determined with $o$-nitrophenyl- $\beta$-D-galactopyranoside discs (Nissui) containing $\sigma$-nitrophenyl- $\beta$-D-galactopyranoside, peptone, and dipotassium hydrogen phosphate in $2 \%$ (wt/vol) $\mathrm{NaCl}$ after $12 \mathrm{~h}$ of incubation. Gelatin hydrolysis was determined in ZoBell's $2216 \mathrm{e}$ broth containing $20 \%$ (wt/vol) gelatin; preparations were observed for liquefaction for 7 days. ZoBell's 2216e agar containing $0.1 \%$ (wt/vol) soluble starch was flooded with Lugol's solution for Gram staining after 2 and 5 days of incubation. The formation of clear zones under and around colonies was recorded as a positive result. ZoBell's $2216 \mathrm{e}$ agar media containing $1 \%$ (wt/vol) sodium alginate, $1 \%(\mathrm{wt} / \mathrm{vol})$ colloidal chitin prepared as described by West and Colwell (34), or $1 \%$ (wt/vol) Tween 80 were used to determine hydrolysis of alginate, chitin, and Tween 80 . Alginase activity was determined after a preparation was overlaid with ethanol after 7 days of incubation. Hydrolysis of alginate and hydrolysis of chitin were indicated by clear zones around colonies. 
TABLE 1. Levels of DNA relatedness between $V$. penaeicida $\mathrm{KH}-1^{\mathrm{T}}$ and other $V$. penaeicida strains and strains belonging to other Vibrio species and related genera

\begin{tabular}{|c|c|c|c|}
\hline Species & Strain $^{a}$ & Origin or source of strain ${ }^{a}$ & $\begin{array}{l}\text { \% DNA reassociation } \\
\text { with strain } \mathrm{KH}-1^{\mathrm{T}}\end{array}$ \\
\hline \multirow[t]{6}{*}{ Vibrio penaeicida } & $\mathrm{KH}-1^{\mathrm{T}}\left(=\mathrm{IFO} 15640^{\mathrm{T}}=\mathrm{JCM} 9123^{\mathrm{T}}\right)^{b}$ & Kuruma prawn, Kagoshima Prefecture & 100 \\
\hline & KA-13 $(=\text { IFO } 15641=\text { JCM 9125 })^{b}$ & Kuruma prawn, Yamaguchi Prefecture & 97 \\
\hline & KO-1 (= JCM 9194) $)^{b}$ & Kuruma prawn, Oita Prefecture & 97 \\
\hline & PO-1 (= JCM 9193) & Kuruma prawn, Hiroshima Prefecture & 87 \\
\hline & PD-A $(=$ IFO $15642=$ JCM 9124) & Kuruma prawn, Hiroshima Prefecture & 90 \\
\hline & ESV-8701 (= JCM 9192) & Kuruma prawn, Ehime Prefecture & 99 \\
\hline V. aestuarianus & ATCC $35048^{\mathrm{T}}$ & ATCC & 10 \\
\hline$V$. alginolyticus & HUFP $9107^{\mathrm{T}}\left(=\right.$ ATCC $\left.17749^{\mathrm{T}}\right)$ & U. Simidu & 14 \\
\hline V. anguillarum & HUFP $5001^{\mathrm{T}}\left(=\right.$ ATCC $\left.19264^{\mathrm{T}}\right)$ & OSU & 10 \\
\hline V. campbellii & HUFP $9109^{\mathrm{T}}\left(=\right.$ ATCC $\left.25920^{\mathrm{T}}\right)$ & U. Simidu & 14 \\
\hline V. carchariae & HUFP $9110^{\mathrm{T}}\left(=\right.$ ATCC $\left.35084^{\mathrm{T}}\right)$ & U. Simidu & 10 \\
\hline V. cholerae & HUFP $5004(=$ NIH 35A3) & M. Nishibuchi & 6 \\
\hline V. cincinnatiensis & ATCC $35912^{\mathrm{T}}$ & ATCC & 8 \\
\hline V. damsela & ATCC $33539^{\mathrm{T}}$ & ATCC & 4 \\
\hline V. diazotrophicus & HUFP $9301^{\mathrm{T}}\left(=\operatorname{ATCC} 33466^{\mathrm{T}}\right)$ & H. Kawasaki & 8 \\
\hline V. fischeri & ATCC $7744^{\mathrm{T}}$ & ATCC & 5 \\
\hline V. fluvialis & HUFP $9302^{\mathrm{T}}\left(=\operatorname{NCTC} 11327^{\mathrm{T}}\right)$ & H. Kawasaki & 9 \\
\hline$V$. gazogenes & HUFP $9303^{\mathrm{T}}\left(=\right.$ ATCC $\left.29988^{\mathrm{T}}\right)$ & H. Kawasaki & 6 \\
\hline$V$. harveyi & HUFP $9111^{\mathrm{T}}\left(=\right.$ ATCC $\left.14126^{\mathrm{T}}\right)$ & U. Simidu & 14 \\
\hline V. logei & HUFP $9310^{\mathrm{T}}\left(=\right.$ ATCC $\left.15382^{\mathrm{T}}\right)$ & H. Kawasaki & 7 \\
\hline$V$. mediterranei & ATCC $4331^{\mathrm{T}}$ & ATCC & 4 \\
\hline$V$. natriegens & ATCC $14048^{\mathrm{T}}$ & ATCC & 9 \\
\hline$V$. navarrensis & ATCC $51183^{\mathrm{T}}$ & ATCC & 10 \\
\hline$V$. nereis & HUFP $9112^{\mathrm{T}}\left(=\right.$ ATCC $\left.25917^{\mathrm{T}}\right)$ & U. Simidu & 12 \\
\hline V. nigripulchritudo & ATCC $27043^{T}$ & ATCC & 18 \\
\hline$V$ ordalii & ATCC $33509^{\mathrm{T}}$ & ATCC & 10 \\
\hline V. orientalis & ATCC $33934^{\mathrm{T}}$ & ATCC & 15 \\
\hline V. parahaemolyticus & HUFP $9114^{\mathrm{T}}\left(=\operatorname{ATCC} 17802^{\mathrm{T}}\right)$ & U. Simidu & 13 \\
\hline V. pelagius & HUFP $9115^{\mathrm{T}}\left(=\right.$ ATCC $\left.25916^{\mathrm{T}}\right)$ & U. Simidu & 12 \\
\hline V. proteolyticus & HUFP $9307^{\mathrm{T}}\left(=\right.$ NCMB $\left.1326^{\mathrm{T}}\right)$ & H. Kawasaki & 6 \\
\hline V. salmonicida & ATCC $43839^{\mathrm{T}}$ & ATCC & 5 \\
\hline$V$. splendidus & HUFP $9117^{\mathrm{T}}\left(=\operatorname{ATCC} 33125^{\mathrm{T}}\right)$ & U. Simidu & 11 \\
\hline V. tubiashii & HUFP $9118^{\mathrm{T}}\left(=\right.$ ATCC $\left.19109^{\mathrm{T}}\right)$ & U. Simidu & 11 \\
\hline V. vulnificus & HUFP $5002^{\mathrm{T}}\left(=\right.$ ATCC $\left.27562^{\mathrm{T}}\right)$ & M. Nishibuchi & 13 \\
\hline Photobacterium phosphoreum & HUFP $9311^{\mathrm{T}}\left(=\right.$ ATCC $\left.11040^{\mathrm{T}}\right)$ & H. Kawasaki & 6 \\
\hline Escherichia coli & $\mathrm{K}-12$ & & 4 \\
\hline
\end{tabular}

${ }^{a}$ ATCC, American Type Culture Collection, Rockville, Md.; HUFP, Hiroshima University Fish Pathology Laboratory Culture Collection, Higashihiroshima-shi, Hiroshima, Japan; IFO, Institute for Fermentation Osaka, Osaka, Japan; JCM, Japan Collection of Microorganisms, The Institute of Physical and Chemical Research (RIKEN), Wako-shi, Saitama, Japan; NCTC, National Collection of Type Cultures, London, England; OSU, Oregon State University, Corvallis.

${ }^{b}$ Strains $\mathrm{KH}-1^{\mathrm{T}}$, KA-13, and $\mathrm{KO}-1$ were used in $\mathrm{G}+\mathrm{C}$ content determinations.

Tween 80 hydrolysis was detected by the presence of turbidity around colonies Hydrolysis of chitin and hydrolysis of Tween 80 were observed daily for 7 days. Accumulation of poly- $\beta$-hydroxybutyrate in cells was determined by observing cells with a phase-contrast microscope daily for 4 days as described by Baumann et al. (4). Growth at different temperatures was determined in $1 \%$ (wt/vol) Bacto Peptone (Difco) ( $\mathrm{pH} \mathrm{7.5)} \mathrm{after} 14$ days of incubation. The range of $\mathrm{NaCl}$ concentrations at which organisms grew was determined in $1 \%(\mathrm{wt} / \mathrm{vol})$ Bacto Peptone (Difco) containing different concentrations of $\mathrm{NaCl}$ after 7 days. Semisolid basal medium (Eiken) was used for acid and gas production tests. Each sugar solution was autoclaved separately and added to the basal medium at a concentration of $1 \%(\mathrm{wt} / \mathrm{vol})$. The cultures were observed every day for 7 days. Simmons medium (Eiken) was used for the citrate utilization test. Utilization of carbon sources was determined in the basal medium of Baumann et al. (4) by using 96-well microplates. Bacterial cell suspensions in basal medium were added to filter-sterilized carbon compounds, and the preparations were placed in wells. Each well contained $200 \mu \mathrm{l}$ of basal medium containing $0.1 \%$ (wt/vol) carbohydrate and about $10^{3}$ organisms, and growth was examined visually for 7 days.

The chemicals used in this study were obtained from Nacalai Tesque, Inc., Kyoto, Japan, unless indicated otherwise.

Determination of DNA base composition and DNA-DNA hybridization. DNAs were extracted from cells grown at $25^{\circ} \mathrm{C}$ for 1 day on heart infusion agar (Difco) prepared with $50 \%$ seawater and were purified by using the methods described by Saito and Miura (27) and Marmur (23). When we used the method of Marmur, we modified it slightly by mixing the chloroform-isoamyl alcohol reagent with an equal volume of phenol before use. $G+C$ contents of DNAs were determined by the high-performance liquid chromatography method described by Kumagai et al. (19). DNA-DNA hybridization was performed by the membrane filter method as described previously (2). Briefly, reference DNA was labeled with tritium by nick translation, using a nick translation kit (Takara Shuzo Co., Ltd., Kyoto, Japan). About $10 \mu \mathrm{g}$ (the exact amount used was recorded) of unlabeled single-stranded DNA immobilized on a nitrocellulose membrane and $0.1 \mu \mathrm{g}$ of labeled reference DNA were reassociated in $0.1 \%$ sodium dodecyl sulfate- $2 \times \mathrm{SSC}(1 \times \mathrm{SSC}$ is $0.15 \mathrm{M} \mathrm{NaCl}$ plus $0.015 \mathrm{M}$ sodium citrate). After incubation for $40 \mathrm{~h}$ at $62^{\circ} \mathrm{C}$, the filters were washed and dried. The radioactivity was measured with an Aloca model LSC-3500E liquid scintillation counter.

\section{RESULTS AND DISCUSSION}

The strains which were tentatively identified as members of Vibrio species in a previous study (12) were gram-negative, facultatively anaerobic, straight or slightly curved rod-shaped organisms. Characteristics of the six strains studied, including type strain $\mathrm{KH}-1$, are shown in Table 2 . We observed a single polar flagellum and no lateral flagella under the conditions which we used (Fig. 1). The strains were oxidase positive and required $\mathrm{NaCl}$ to grow. The $\mathrm{G}+\mathrm{C}$ contents of three representative strains were quite similar, ranging from 46.2 to 47.0 mol\%; the $\mathrm{G}+\mathrm{C}$ content of strain $\mathrm{KH}-1^{\mathrm{T}}(\mathrm{T}=$ type strain) was $47.0 \mathrm{~mol} \%$. The strains were susceptible to $\mathrm{O} / 129$ (inhibition zones were 2 to $3 \mathrm{~mm}$ wide when $10-\mu \mathrm{g}$ discs were used). On the basis of the characteristics described above, we confirmed 
TABLE 2. Biochemical and physiological characteristics of six $V$. penaeicida strains $^{a}$

\begin{tabular}{|c|c|c|}
\hline Characteristic & $\begin{array}{l}\% \text { of strains } \\
\text { positive }\end{array}$ & $\begin{array}{l}\text { Reaction of } \\
\text { strain KH-1 }\end{array}$ \\
\hline Fermentation of glucose ( 4 days) & 100 & $t^{b}$ \\
\hline Gas produced from glucose (4 days) & 0 & - \\
\hline Oxidase & 100 & + \\
\hline Catalase & 100 & + \\
\hline Indole production ( 5 days) & 50 & - \\
\hline Nitrate reduction ( 2 days) & 100 & + \\
\hline Voges-Proskauer test ( 5 days) & 0 & - \\
\hline Hydrogen sulfide production ( 7 days) & 0 & - \\
\hline Arginine dihydrolase ( 7 days) & 0 & - \\
\hline Lysine decarboxylase ( 7 days) & 0 & - \\
\hline Ornithine decarboxylase (7 days) & 0 & - \\
\hline ONPG hydrolysis ${ }^{c}$ & 100 & + \\
\hline Alginase ( 7 days) & 17 & + \\
\hline Amylase ( 2 days) & 83 & + \\
\hline Chitinase ( 7 days) & 100 & + \\
\hline Gelatinase ( 3 days) & 100 & + \\
\hline Lipase ( 3 days) & 100 & + \\
\hline PHB accumulation (4 days) $)^{d}$ & 0 & - \\
\hline \multicolumn{3}{|l|}{ Growth at: } \\
\hline $4^{\circ} \mathrm{C}$ & 0 & - \\
\hline $20^{\circ} \mathrm{C}$ & 100 & + \\
\hline $30^{\circ} \mathrm{C}$ & 100 & + \\
\hline $35^{\circ} \mathrm{C}$ & 0 & - \\
\hline $40^{\circ} \mathrm{C}$ & 0 & - \\
\hline \multicolumn{3}{|l|}{ Growth in the presence of: } \\
\hline $0 \% \mathrm{NaCl}$ & 0 & - \\
\hline $0.5 \% \mathrm{NaCl}$ & 0 & - \\
\hline $1 \% \mathrm{NaCl}$ & 100 & + \\
\hline $3 \% \mathrm{NaCl}$ & 100 & + \\
\hline $5 \% \mathrm{NaCl}$ & 50 & + \\
\hline $6 \% \mathrm{NaCl}$ & 0 & - \\
\hline \multicolumn{3}{|l|}{ Acid production ( 7 days) from: } \\
\hline Adonitol & 0 & - \\
\hline L-Arabinose & 0 & - \\
\hline D-Cellobiose & 100 & + \\
\hline Dextrin & 100 & + \\
\hline Dulcitol & 0 & - \\
\hline Fructose & 100 & + \\
\hline D-Galactose & 0 & - \\
\hline D-Glucose & 100 & + \\
\hline Glycerol & 0 & - \\
\hline Glycogen & 50 & + \\
\hline myo-Inositol & 0 & - \\
\hline Lactose & 50 & - \\
\hline Maltose & 100 & + \\
\hline D-Mannitol & 0 & - \\
\hline D-Mannose & 100 & + \\
\hline Melibiose & 0 & - \\
\hline Raffinose & 0 & - \\
\hline L-Rhamnose & 0 & - \\
\hline Salicin & 0 & - \\
\hline D-Sorbitol & 0 & - \\
\hline Sucrose & 0 & - \\
\hline Trehalose & 100 & + \\
\hline D-Xylose & 0 & - \\
\hline
\end{tabular}

Continued

that Vibrio species PJ belongs to the genus Vibrio. Although there were some variations in physiological and biochemical characteristics among the six strains of Vibrio species PJ (Table 2), all of the strains were distinct from other Vibrio species (Table 3). The levels of DNA relatedness between five strains of Vibrio species PJ and type strain $\mathrm{KH}-1$ were $87 \%$ or more; this indicated that the strains which we studied are closely related (Table 1 ). The levels of DNA relatedness between
TABLE 2-Continued

\begin{tabular}{|c|c|c|}
\hline Characteristic & $\begin{array}{l}\% \text { of strains } \\
\text { positive }\end{array}$ & $\begin{array}{l}\text { Reaction of } \\
\text { strain KH-1 }\end{array}$ \\
\hline \multicolumn{3}{|c|}{$\begin{array}{l}\text { Utilization as a sole carbon source } \\
\text { ( } 7 \text { days) of: }\end{array}$} \\
\hline$\gamma$-Aminobutyrate & 33 & - \\
\hline D-Cellobiose & 100 & + \\
\hline Citrate (Simmons) & 67 & + \\
\hline L-Citrulline & 33 & - \\
\hline Ethanol & 0 & - \\
\hline D-Gluconate & 83 & + \\
\hline D-Glucuronate & 0 & - \\
\hline$\beta$-Hydroxybutyrate & 83 & + \\
\hline L-Leucine & 0 & - \\
\hline DL-Malate & 100 & + \\
\hline D-Mannitol & 83 & + \\
\hline Propionate & 0 & - \\
\hline Putrescine & 0 & - \\
\hline Sucrose & 0 & - \\
\hline D-Xylose & 0 & - \\
\hline
\end{tabular}

${ }^{a}$ All tests except the temperature range test were performed at $25^{\circ} \mathrm{C}$.

$b+$, positive; - , negative.

${ }^{c}$ ONPG, $o$-nitrophenyl- $\beta$-D-galactopyranoside.

${ }^{d}$ PHB, poly- $\beta$-hydroxybutyrate.

strain $\mathrm{KH}-1^{\mathbf{T}}$ and type or reference strains of 28 previously described Vibrio species were significantly lower (less than $18 \%$ ).

The following seven species were not used in DNA-DNA hybridization experiments: Vibrio costicola, Vibrio furnissii, Vibrio hollisae, Vibrio marinus, Vibrio metschnikovii, Vibrio mimicus, and Vibrio mytili. V. costicola, V. furnissii, V. hollisae, and $V$. marinus differ from the prawn pathogen in their DNA $\mathrm{G}+\mathrm{C}$ contents and phenotypic characteristics. In addition to the characteristics shown in Table 3 , these organisms could be differentiated by the following test results: $V$. furnissii produces gas from $\mathrm{D}$-glucose and acid from L-arabinose and uses ethanol and putrescine (5); $V$. hollisae produces acid from L-arabinose (17); and $V$. marinus can grow at 4 and $30^{\circ} \mathrm{C}(5)$. It has been reported that the type strain of $V$. mimicus (ATCC 33653) exhibited a high level of DNA relatedness to the type strain of $V$. cholerae $(67 \%)(10)$. However, the representative strain of the prawn pathogen, type strain KH-1, exhibited only $6 \%$ DNA relatedness to $V$. cholerae HUFP 5004, which is closely related genetically to the type strain of $V$. cholerae (see above). Therefore, V. mimicus and the prawn pathogen can be differentiated from each other genetically. The remaining two species not used in DNA-DNA hybridization experiments could be distinguished clearly from the prawn isolates on the basis of phenotypic characteristics (Table 3). In addition, $V$. metschnikovii is Voges-Proskauer positive and negative for oxidase activity and nitrate reduction (5); and V. mytili pro-

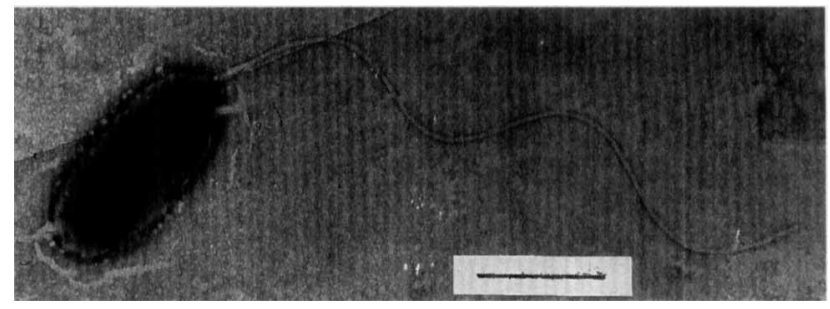

FIG. 1. Electron micrograph of a negatively stained cell of $V$. penaeicida $\mathrm{KH}-1^{\mathrm{T}}$, showing the single polar flagellum. Bar $=1 \mu \mathrm{m}$. 
TABLE 3. Characteristics useful for distinguishing V. penaeicida from previously described Vibrio species ${ }^{a}$

\begin{tabular}{|c|c|c|c|c|c|c|c|c|c|c|c|c|c|}
\hline \multirow{2}{*}{ Taxon } & \multirow{2}{*}{$\begin{array}{c}\text { Suscepti- } \\
\text { bility to } \\
\mathrm{O} / 129 \\
(10 \mu \mathrm{g})\end{array}$} & \multirow{2}{*}{$\begin{array}{l}\text { Arginine } \\
\text { dihydro- } \\
\text { lase }\end{array}$} & \multirow{2}{*}{$\begin{array}{l}\text { Lysine } \\
\text { decarbox- } \\
\text { ylase }\end{array}$} & \multirow{2}{*}{$\begin{array}{l}\text { Ornithine } \\
\text { decarbox- } \\
\text { ylase }\end{array}$} & \multirow{2}{*}{$\begin{array}{l}\text { Growth } \\
\text { in the } \\
\text { presence of } \\
6 \% \mathrm{NaCl}\end{array}$} & \multirow{2}{*}{$\begin{array}{c}\text { Growth } \\
\text { at } \\
35^{\circ} \mathrm{C}^{b}\end{array}$} & \multirow{2}{*}{$\begin{array}{l}\text { Gelati- } \\
\text { nase }\end{array}$} & \multirow{2}{*}{ Lipase } & \multirow{2}{*}{$\begin{array}{c}\text { Acid } \\
\text { produced } \\
\text { from } \\
\text { sucrose }\end{array}$} & \multirow{2}{*}{$\begin{array}{l}\text { Lumines- } \\
\text { cence }\end{array}$} & \multicolumn{2}{|c|}{ Utilization of $\mathrm{f}^{b}$ : } & \multirow{2}{*}{$\begin{array}{c}\mathrm{G}+\mathrm{C} \\
\text { content } \\
(\mathrm{mol} \%)\end{array}$} \\
\hline & & & & & & & & & & & $\begin{array}{l}\text { D-Glucu- } \\
\text { ronate }\end{array}$ & $\begin{array}{l}\text { Propio- } \\
\text { nate }\end{array}$ & \\
\hline Vibrio penaeicida & $+^{d}$ & - & - & - & - & - & + & + & - & - & - & - & $46-47$ \\
\hline V. aestuarianus ${ }^{e}$ & + & + & - & - & ND & + & + & ND & + & - & ND & ND & $43-44$ \\
\hline V. alginolyticus & - & - & + & + & + & + & + & + & + & - & - & + & $45-47$ \\
\hline$V$. anguillarum & + & + & - & - & + & + & + & + & + & - & - & + & $44-46$ \\
\hline V. campbellii & - & - & + & - & + & + & + & + & - & - & - & + & $46-48$ \\
\hline V. carchariae $^{f}$ & - & - & + & + & + & + & + & + & + & - & ND & - & 46 \\
\hline V. cholerae & + & - & + & + & $\mathrm{v}$ & + & + & + & + & $\mathrm{v}$ & - & + & $47-49$ \\
\hline V. cincinnatiensis ${ }^{g}$ & - & - & + & - & + & + & - & - & + & - & - & ND & 45 \\
\hline V. costicola & - & + & - & - & + & + & + & $\mathrm{v}$ & + & - & - & + & 50 \\
\hline$V$. damsela $^{h}$ & ND & + & - & - & + & + & - & - & - & - & - & - & 43 \\
\hline V. diazotrophicus ${ }^{i}$ & ND & + & - & - & + & + & - & - & + & - & + & v & $46-47$ \\
\hline V. fischeri & + & - & + & - & + & $\mathrm{v}$ & - & + & - & + & - & - & $39-41$ \\
\hline V. fluvialis & - & + & - & - & + & + & + & + & + & - & + & + & $49-51$ \\
\hline V. furnissii & - & + & - & - & + & + & + & + & + & - & - & + & 50 \\
\hline V. gazogenes & + & - & - & - & + & + & + & + & + & - & - & + & 47 \\
\hline V. harveyi & - & - & + & + & + & + & + & + & $\mathrm{v}$ & $\mathrm{v}$ & + & + & $46-48$ \\
\hline V. hollisae ${ }^{j}$ & ND & - & - & - & $\mathrm{v}$ & + & - & - & - & - & ND & ND & 50 \\
\hline V. logei & $\mathrm{v}$ & - & + & - & ND & - & - & $\mathrm{v}$ & $\mathrm{v}$ & + & - & - & $40-42$ \\
\hline$V$. marinus & - & - & + & - & - & - & $\mathrm{v}$ & + & $\mathrm{v}$ & - & - & - & 42 \\
\hline$V$. mediterranei $^{k}$ & ND & - & $\mathrm{v}$ & - & ND & ND & - & + & + & $\mathrm{v}$ & ND & ND & $42-43$ \\
\hline V. metschnikovii & + & + & $\mathrm{v}$ & - & + & + & + & + & + & - & - & - & $44-46$ \\
\hline V. mimicus ${ }^{l}$ & $\mathrm{v}$ & - & + & + & $\mathrm{v}$ & + & + & + & $\mathrm{v}$ & - & + & - & ND \\
\hline$V \cdot$ mytilim $^{m}$ & ND & + & - & - & + & + & - & + & + & - & - & ND & $45-46$ \\
\hline$V$. natriegens & - & - & - & - & + & + & + & + & + & & $\mathrm{v}$ & + & $46-47$ \\
\hline V. navarrensis ${ }^{n}$ & ND & - & - & - & + & + & + & ND & + & - & - & - & $45-47$ \\
\hline$V$. nereis & - & + & - & - & + & + & $\mathbf{v}$ & - & + & - & - & + & $46-47$ \\
\hline V. nigripulchritudo & - & - & - & - & - & - & + & + & - & - & + & + & $46-47$ \\
\hline V. ordalii & + & - & - & - & + & - & + & - & + & - & - & - & $44-46$ \\
\hline V. orientalis ${ }^{o}$ & ND & + & ND & ND & ND & + & + & + & ND & + & - & - & $45-46$ \\
\hline V. parahaemolyticus & - & - & + & + & + & + & + & + & - & - & $\mathrm{v}$ & + & $46-47$ \\
\hline$V$ pelagius biovar I & + & - & - & - & + & + & - & + & + & - & - & + & $45-47$ \\
\hline V. pelagius biovar II & + & - & - & - & + & + & + & + & + & - & - & + & $45-47$ \\
\hline V. proteolyticus & - & + & + & - & + & + & + & + & - & - & - & - & 51 \\
\hline V. salmonicida ${ }^{p}$ & ND & - & ND & ND & - & - & - & - & ND & ND & ND & ND & 42 \\
\hline V. splendidus biovar I & + & + & - & - & $\mathrm{v}$ & $\mathrm{v}$ & + & + & $\mathrm{v}$ & + & + & + & $45-46$ \\
\hline V. splendidus biovar II & + & - & - & - & $\mathrm{v}$ & - & + & + & - & - & - & + & $45-46$ \\
\hline V. tubiashii ${ }^{q}$ & $\mathrm{v}$ & + & - & - & $\mathrm{v}$ & + & + & + & + & - & - & - & $43-45$ \\
\hline V. vulnificus biovar I & + & - & + & + & + & + & + & + & - & - & + & + & $46-48$ \\
\hline V. vulnificus biovar $\mathbf{I I}^{\mathrm{r}}$ & ND & - & + & + & ND & + & + & + & - & - & ND & ND & $46-47$ \\
\hline
\end{tabular}

${ }^{a}$ Data from reference 34 , except as indicated below.

${ }^{b}$ Data from reference 18 .

Data from reference 5 .

${ }^{d}+$, positive for at least $90 \%$ of the strains; - , negative for at least $90 \%$ of the strains; v, variable among strains; ND, no data available.

${ }^{e}$ Data from reference 31.

${ }^{f}$ Data from reference 14

${ }^{g}$ Data from reference 6 .

${ }^{h}$ Data from reference 21.

${ }^{i}$ Data from reference 15

${ }^{j}$ Data from reference 17 .

${ }^{k}$ Data from reference 24.

${ }^{l}$ Data from reference 33.

${ }^{m}$ Data from reference 25 .

${ }^{n}$ Data from reference 32.

${ }^{\circ}$ Data from reference 35.

${ }^{P}$ Data from reference 13 .

q Data from reference 16.

${ }^{r}$ Data from reference 30.

duces acid from L-arabinose, D-galactose, and D-xylose and gas from D-glucose (25).

$V$. penaeicida can be differentiated from 35 other Vibrio species, even species with similar $\mathrm{G}+\mathrm{C}$ contents, by the following characteristics: arginine dihydrolase activity, lysine and ornithine decarboxylase activities, growth in the presence of $6 \% \mathrm{NaCl}$ and at $35^{\circ} \mathrm{C}$, production of acid from sucrose, utilization of $\mathrm{D}$-glucuronate and propionate, and luminescence
(Table 3). In addition to the differential features shown in Table 3, other Vibrio species can be differentiated from $V$. penaeicida as follows: Vibrio mediterranei produces acid from D-mannitol and D-sorbitol (24); Vibrio nigripulchritudo produces a black pigment (5); Vibrio salmonicida is negative for production of indole and chitinase and nitrate reduction (13); and Vibrio splendidus biovar II does not produce acid from D-mannose (5). 
On the basis of the results described above, we propose the name Vibrio penaeicida sp. nov. for Vibrio species PJ.

Description of Vibrio penaeicida sp. nov. Vibrio penaeicida (pe.nae.i.ci'da. L. n. Penaeus, genus name of kuruma prawns; L. v. caedo, to kill; L. adj. penaeicida, Penaeus killer) cells are gram-negative short rods ( 1.5 to 2 by 0.5 to $0.8 \mu \mathrm{m}$ ) that are straight or slightly curved and motile by means of a single polar flagellum when the cells are grown on solid media. No lateral flagella are formed on solid media, and there is no swarming. Does not form spores. Facultatively anaerobic. Susceptible to 2,4-diamino-6,7-diisopropylpteridine (vibriostatic agent $\mathrm{O} / 129$ ) at a concentration of $10 \mu \mathrm{g}$ per disc.

After incubation for $24 \mathrm{~h}$ at $25^{\circ} \mathrm{C}$ colonies on ZoBell's $2216 \mathrm{e}$ agar are low convex, entire, circular, cream colored, and translucent. Neither pigmentation nor luminescence is observed. Biochemical and physiological characteristics are shown in Table 2.

The $\mathrm{G}+\mathrm{C}$ contents of the DNAs range from 46.2 to 47.0 mol\% in the three strains that have been tested.

It has been demonstrated that there is a major common $\mathrm{O}$ antigen in all six strains tested (12). $V$. penaeicida can be differentiated serologically from fish-pathogenic vibrios such as Vibrio anguillarum (nine serovars), Vibrio alginolyticus, $V$. cholerae (non-O:1), Vibrio ordalii, and Vibrio vulnificus (12).

$V$. penaeicida is the causative agent of vibriosis of kuruma prawns in Japan (12).

The type strain is strain KH-1 (=IFO $15640=$ JCM 9123).

\section{ACKNOWLEDGMENTS}

We thank Usio Simidu of Hiroshima University, Hiroko Kawasaki of the Institute of Applied Microbiology, The University of Tokyo, and the staff of several prefectural fishery experiment stations for supplying bacterial strains.

\section{REFERENCES}

1. Aaronson, S. 1970. Experimental microbial ecology, p. 70. Academic Press, New York.

2. Akagawa-Matsushita, M., M. Matsuo, Y. Koga, and K. Yamasato. 1992. Alteromonas atlantica sp. nov. and Alteromonas carrageenovora sp. nov., bacteria that decompose algal polysaccharides. Int. J. Syst. Bacteriol. 42:621627.

3. Allen, R. D., and P. Baumann. 1971. Structure and arrangement of flagella in species of the genus Beneckea and Photobacterium fischeri. J. Bacteriol. 107:295-302.

4. Baumann, P., L. Baumann, and M. Mandel. 1971. Taxonomy of marine bacteria: the genus Beneckea. J. Bacteriol. 107:268-294.

5. Baumann, P., and R. H. W. Schubert. 1984. Genus I. Vibrio, p. 518-538. In N. R. Krieg and J. G. Holt (ed.), Bergey's manual of systemematic bacteriology, vol. 1. The Williams \& Wilkins Co., Baltimore.

6. Brayton, P. R., R. B. Bode, R. R. Colwell, M. T. MacDonell, H. L. Hall, D. J. Grimes, P. A. West, and T. N. Brayant. 1986. Vibrio cincinnatiensis sp. nov., a new human pathogen. J. Clin. Microbiol. 23:104-108.

7. Citarella, R. V., and R. R. Colwell. 1970. Polyphasic taxonomy of the genus Vibrio: polynucleotide sequence relationships among selected Vibrio species. J. Bacteriol. 104:434-442.

8. Conn, H. J., J. W. Bartholomew, and M. W. Jennison. 1957. Staining methods, p. 10-36. In Committee on Bacteriological Technic of the Society of American Bacteriologists (ed.), Manual of microbiological methods. McGraw-Hill Book Co., New York.

9. Conn, H. J., M. W. Jennison, and O. B. Weeks. 1957. Routine tests for the identification of bacteria, p. 140-168. In Committee on Bacteriological Technic of the Society of American Bacteriologists (ed.), Manual of microbiological methods. McGraw-Hill Book Co., New York.
10. Davis, B. R., G. R. Fanning, J. M. Madden, A. G. Steigerwalt, H. B. Bradford, Jr., H. L. Smith, Jr., and D. J. Brenner. 1981. Characterization of biochemically atypical Vibrio cholerae strains and designation of a new pathogenic species, Vibrio mimicus. J. Clin. Microbiol. 14:631-639.

11. de la Peña, L. D., K. Momoyama, T. Nakai, and K. Muroga. 1992. Detection of the causative bacterium of vibriosis in kuruma prawn, Penaeus japonicus. Fish Pathol, 27:223-228.

12. de la Peña, L. D., T. Tamaki, K. Momoyama, T. Nakai, and K. Muroga. 1993. Characteristics of the causative bacterium of vibriosis in kuruma prawn, Penaeus japonicus. Aquaculture 115:1-12.

13. Egidius, E., R. Wiik, K. Andersen, K. A. Hoff, and B. Hjeltnes. 1986. Vibrio salmonicida sp. nov., a new fish pathogen. Int. J. Syst. Bacteriol. 36:518-520.

14. Grimes, D. J., J. Stemmler, H. Hada, E. B. May, D. Maneval, F. M. Hetrick, R. T. Jones, M. Stoskopf, and R. R. Colwell. 1984. Vibrio species associated with mortality of sharks held in captivity. Microb. Ecol. 10:271-282.

15. Guerinot, M. L., P. A. West, J. V. Lee, and R. R. Colwell. 1982. Vibrio diazotrophicus sp. nov., a marine nitrogen-fixing bacterium. Int. J. Syst. Bacteriol. 32:350-357.

16. Hada, H. S., P. A. West, J. V. Lee, J. Stemmler, and R. R. Colwell. 1984. Vibrio tubiashii sp. nov., a pathogen of bivalve mollusks. Int. J. Syst. Bacteriol. 34:1-4.

17. Hickman, F. W., J. J. Farmer III, D. G. Hollis, G. R. Fanning, A. G. Steigerwalt, R. E. Weaver, and D. J. Brenner. 1982. Identification of Vibrio hollisae sp. nov. from patients with diarrhea. J. Clin. Microbiol. 15:395-401.

18. Holt, J. G., N. R. Krieg, P. H. A. Sneath, J. T. Staley, and S. T. Williams (ed.). 1994. Bergey's manual of determinative bacteriology, 9th ed., p. 192-194, 260-274. The Williams \& Wilkins Co., Baltimore.

19. Kumagai, M., M. Fujimoto, and A. Kuninaka. 1988. Determination of base composition of DNA by high performance liquid chromatography of its nuclease P1 hydrolysate. Nucleic Acids Res. Symp. Ser. 19:65-68.

20. Lee, J. V., M. S. Hendrie, and J. M. Shewan. 1979. Identification of Aeromonas, Vibrio and related organisms, p. 151-166. In F. A. Skinner and D. W. Lovelock (ed.), Identification methods for microbiologists, 2nd ed. Academic Press, London.

21. Love, M., D. Teebken-Fisher, J. E. Hose, J. J. Farmer III, F. W. Hickman, and G. R. Fanning. 1981. Vibrio damsela, a marine bacterium, causes skin ulcers on the damselfish Chromis punctipinnis. Science 214:1139-1140.

22. MacFaddin, J. F. 1980. Biochemical tests for identification of medical bacteria, 2nd ed. Williams \& Wilkins Co., Baltimore.

23. Marmur, J. 1961. A procedure for the isolation of deoxyribonucleic acid from microorganisms. J. Mol. Biol. 3:208-218.

24. Pujalte, M. J., and E. Garay. 1986. Proposal of Vibrio mediterranei sp. nov.: a new marine member of the genus Vibrio. Int. J. Syst. Bacteriol. 36:278-281.

25. Pujalte, M. J., M. Ortigosa, M. C. Urdaci, E. Garay, and P. A. D. Grimont 1993. Vibrio mytili sp. nov., from mussels. Int. J. Syst. Bacteriol. 43:358-362.

26. Reichelt, J. L., and P. Baumann. 1973. Taxonomy of the marine, luminous bacteria. Arch. Mikrobiol. 94:283-330.

27. Saito, H., and K. Miura. 1963. Preparation of transforming deoxyribonucleic acid by phenol treatment. Biochim. Biophys. Acta 72:619-629.

28. Sano, T., and H. Fukuda. 1987. Principal microbial diseases of mariculture in Japan. Aquaculture 67:59-69.

29. Takahashi, Y., Y. Shimoyama, and K. Momoyama. 1985. Pathogenicity and characteristics of Vibrio sp. isolated from cultured kuruma prawn Penaeus japonicus Bate. Bull. Jpn. Soc. Sci. Fish. 51:721-730.

30. Tison, D. L., M. Nishibuchi, J. D. Greenwood, and R. J. Seidler. 1982. Vibrio vulnificus biogroup 2: new biogroup pathogenic for eels. Appl. Environ. Microbiol. 44:640-646.

31. Tison, D. L., and R. J. Seidler. 1983. Vibrio aestuarianus: a new species from estuarine waters and shellfish. Int. J. Syst. Bacteriol. 33:699-702.

32. Urdaci, M. C., M. Marchand, E. Ageron, J. M. Arcos, B. Sesma, and P. A. D. Grimont. 1991. Vibrio navarrensis sp. nov., a species from sewage. Int. J. Syst. Bacteriol. 41:290-294.

33. West, P. A., P. R. Brayton, T. N. Bryant, and R. R. Colwell. 1986. Numerical taxonomy of vibrios isolated from aquatic environments. Int. J. Syst. Bacteriol. 36:531-543.

34. West, P. A., and R. R. Colwell. 1984. Identification and classification of Vibrionaceae - an overview, p. 285-363. In R. R. Colwell (ed.), Vibrios in the environment. John Wiley \& Sons, Inc., New York.

35. Yang, Y., L. P. Yah, Y. Cao, L. Baumann, P. Baumann, J. S. Tang, and B. Beaman. 1983. Characterization of marine luminous bacteria isolated off the coast of China and description of Vibrio orientalis sp. nov. Curr. Microbiol. 8:95-100. 